\title{
PARALLAX PROBLEMS OLD AND NEW
}

\author{
BY JAN SCHILT
}

\begin{abstract}
The systematic corrections to the various parallax series used in the new Parallax Catalogue should probably not be applied. The average of these corrections over the entire sky is negative and if they are applied the result is that the average absolute parallax, at least for the southern stars, comes out smaller than the observed relative one. The printed absolute parallaxes would thus require corrections up to +".oo7.

It is found from an as yet limited material that the relative parallaxes depend on the mean magnitude of the comparison stars. If this is confirmed the mean parallax of the I I mag. (pg) stars used as comparison stars must in many cases be very much larger than the statistical value derived from proper motions.
\end{abstract}

The critical examination of the errors in trigonometric parallaxes is complicated by the varied nature of most observing programs and the preferential treatment of certain classes of stars. This, in general, upsets the error distribution and the mean values, and it may produce spurious systematic differences in the case of comparisons between different stations. The classical case in point is the rejection of negative parallaxes to which practice Kapteyn first drew attention. In the same category is the addition of plates in the case of a negative parallax. Spurious systematic effects appear if particularly large parallaxes, already observed elsewhere, are repeated, because preference is given to parallaxes with positive accidental errors.

Of interest is the general case that two stations have unequal error distributions. The differences between the parallaxes depend then on their sums, and a regression effect is produced which is especially conspicuous on the side of the small parallaxes. The effect described by Louise Jenkins is probably of this nature. ${ }^{1}$ In the case described, for instance, in Fig. I (upper curve) of the paper just quoted the gradual increase of Y-A from - ".oo6 to +".oro on the left side of the plot would show that the error distribution, at least on the negative side, is wider for Yale than for Allegheny. It may be that the Yale errors for small parallaxes are larger than the Allegheny errors as a consequence of the policy whereby the least interesting stars are left to routine technicians whereas the "plums" are picked out for measurement by the professional astronomers. It is also possible that the difference is due to suppression of negative parallaxes at Allegheny, or to any policy that restricts the dispersion in the relative parallaxes. According to the last paragraph of this paper it may even be the selection of the comparison stars.

In the event that trigonometric minus spectroscopic parallax is plotted against spectroscopic the observed slope will again depend on the dispersions. If the dispersion in the trigonometric parallaxes is small, as e.g. in the case of the $\mathrm{Yk}$ Spec diagram given by Jenkins l.c., the dominant feature may be a slope approaching $-\mathbf{I} .(=\tan$ $135^{\circ}$ ) caused by the errors in the spectroscopic parallaxes. If, however, the dispersion in the trigonometric parallaxes is larger, as in the case $\mathrm{Y}$-Spec, this regression will be buried in a wider distribution of which the slope cannot be predicted without a detailed analysis of the kinds of stars included in the sample. That the trigonometric parallaxes would have errors depending on the size of the parallax is at the outset very unlikely, and it must be concluded that the apparent relations shown in these and similar diagrams depend on the dispersions of the samples; they do not call for systematic corrections.

There remain the constant corrections to the parallaxes of a given observatory. Schlesinger has combined these corrections with the reductions from relative to absolute, but for the present discussion it is better to consider them separately. The mean parallax of the comparison stars ranges from ".002 to ".004 in the magnitude range used by parallax observers. The average values do not vary by more than "oor from one station to another, and are very close to ".oo3. Only the Mt. Wilson parallaxes are excepted. The systematic corrections that have actually been used in the parallax catalogues are thus obtained by subtracting ".003 from the values under the Precepts. Listing only the most important stations the following values are obtained for the I935 and 1952 catalogues:

TABLE I. SYSTEMATIC CORRECTIONS I 935 AND I952

\begin{tabular}{lrr} 
& \multicolumn{1}{c}{ I935 } & \multicolumn{1}{c}{1952} \\
Allegheny & ".000 & ".000 \\
McCormick & .000 & -.001 \\
Yale & -.003 & -.005 \\
Yerkes & .000 & -.005 \\
Mt. Wilson & -.005 & -.004 \\
Greenwich & .000 & -.001 \\
Sproul & -.005 & -.006 \\
Cape & -.001 & -.006
\end{tabular}


The average by stations is - ".oor7 (1935) and -".o035 (I952). These corrections have their origin in the differences between the parallaxes of the same stars observed at two or more stations. The problem is what to do with these differences. Should they be applied with positive sign to the station giving the smaller, or with negative to that giving the larger, or is neither justified? In Schlesinger's solution zero correction was derived for the Allegheny parallaxes, and this value has been adopted in the new catalogue. Extensive use was made in the derivation of Schlesinger's system of trigonometric parallaxes of the Mt. Wilson spectroscopic parallaxes, and scale corrections to the latter were derived in the course of the work. The details of the process of adjustment, and the equations of condition have not been published, but it seems that the condition whereby the differences between trigonometric and spectroscopic parallaxes were minimized has unduly favoured small parallaxes; this follows from the proportionality between the spectroscopic parallaxes and their errors. There is, moreover, no doubt now that the Mt. Wilson spectroscopic parallaxes are seriously in error as to the dispersion in the absolute magnitudes inside a spectral division. Another point that Schlesinger considered was that whereas it seemed possible that an observer would by rejection or deferment of a star cause his published parallaxes to be statistically too large it would be psychologically improbable that they would be too small. This may be questioned, since the realization of the danger of obtaining statistically too large parallaxes through rejection of negative ones may well have caused the contrary effect. Finally, the Allegheny system seemed to be supported at least by the majority of the stations and there were only three that required appreciable corrections, vis. Yale, Sproul, and Mt. Wilson. As seen in Table I, however, this is no longer the case. The results for 1952 show Allegheny in a minority with McCormick and Greenwich whereas the other 5 stations show corrections from -".004 to -".o06. The assumption that Allegheny rather than any of the other stations should be given zero correction is, therefore, rather arbitrary, and the corrections of Table I should not be accepted as absolute. If, as a simple procedure, it is desired to reduce the average correction by stations to zero one has to apply +".0035 to the values given under the precepts on page (4) of the Parallax Catalogue.

The next question to be considered is the reality of the relative systematic corrections. By this the following is meant. Suppose it is found from the stars common to two stations, $\mathrm{Y}$ and $\mathrm{A}$, that $\mathrm{Y}-\mathrm{A}=+$ ".005. This clearly means that the average error in $\mathrm{Y}$ is algebraically ".005 larger than that of $\mathrm{A}$ as far as the parallaxes of the sample are concerned. The question is whether the same difference holds for the parallaxes observed at $\mathrm{Y}$ but not at $\mathrm{A}$, and those at $\mathrm{A}$ but not at $\mathrm{Y}$. In considering this question the following points are of interest. I. It may be mentioned that no satisfactory explanation for the existence of such systematic errors has been put forward. The entire procedure from the taking of the plates to the final reductions has been frequently scrutinized by the experts in the field, and seems to preclude sizable errors. Errors of instrumental origin including magnitude errors have to be of opposite sign for morning and evening observations in order to affect the parallax. The fatigue factor which would cause less accurate guiding in the morning than in the evening with the result that images of the parallax star are displaced relative to the comparison stars in opposite directions mornings and evenings, has been offered as an explanation, but is not a satisfactorily one since the morning observations are generally made by a fresh observer. Orientation of component parts of the lens with respect to each other has been investigated by van de Kamp as a source of systematic errors, ${ }^{2}$ but the resulting effects, though noticeable, are far too small. 2 . In the earlier parallaxes the mean values were affected by omission of negative and otherwise unacceptable results. Although this policy is no longer the rule, it should be expected that some traces of the effect are still present. 3. Comparing the 1935 and the 1952 catalogues, there are two cases, Yerkes and Cape, in which the corrections have undergone notable changes. In the case of the Cape this means better agreement with Yale. These two southern stations have by now a very large number of stars in common, and the smallness of the relative correction, which in this case is mainly based on direct comparisons, is gratifying. In the case of Yerkes no recent observations have been added, and the change is largely an effect of the peculiarities of the least-squares solution. 4. The stars common to two stations are as a rule, even in the absence of personal bias, selected by some quality like magnitude or spectral range, and, naturally, by declination; in general they cannot be a random sample. 5. It 
seems very unlikely that a systematic difference such as $\mathrm{Y}-\mathrm{A}=+$..005 would be entirely due to properties of the stations, and independent of any properties of the stars. It would perhaps seem less improbable that it would be a function of some quality of the stars, and especially one that would be extreme for the stars in common.

On the strength of the available evidence there must be considerable doubt as to the validity of the relative corrections. In some cases the number of stars in common are small, and the mean differences are uncertain. In other cases the differences are probably significant, but as their nature is not understood there is no valid reason to assume that such differences as are found, for example, between Yale and Allegheny should hold outside the region of overlap. Whereas no procedure can be entirely satisfactory until more light is thrown on the question it seems best not to apply the corrections, but to use the relative parallaxes as observed, and to apply $+{ }^{\prime \prime} .003$ for latitudes less than $45^{\circ}$, following the practice of the parallax catalogues, +".oo 4 for $45^{\circ}$ to $70^{\circ}$, and + ".o05 for $70^{\circ}$ to $90^{\circ}$, as the reduction to absolute. Absolute parallaxes have been computed in accordance with the present recommendations, but using the same weights as given in the 1952 Parallax Catalogue for the stars numbered I, 5I, IoI, etc. The changes in the absolute parallaxes range from ".ooo to +".oo7 and apply to the following numbers of stars:

$\begin{array}{cc}\begin{array}{c}\text { Increase } \\ \text { in Abs. Par. }\end{array} & \begin{array}{c}\text { No. of } \\ \text { Stars }\end{array} \\ \text { ".000 } & \text { I7 } \\ +.001 & 20 \\ +.002 & \text { I } \\ +.003 & 8 \\ +.004 & \text { I } \\ +.005 & \text { I } \\ +.006 & 20 \\ + \text { ".007 } & 4 \\ >+ \text { +.007 } & 2\end{array}$

The question whether the parallax of the comparison stars could have something to do with the various discrepancies should be revived. ${ }^{3}$ Large deviations from the mean parallaxes of IIth magnitude stars would be required, and this is possible only if there are clouds of nearby stars. In order to be eligible as comparison stars they would have to be of very small proper motions. The existence of such objects could account for systematic differences between the relative parallaxes by different observers, and also for certain regions where negative parallaxes are predominant, and it might conceivably lead to an explana- tion of some other hitherto unexplained effects. Comparison stars in a nearby cloud will give a small parallax, and if the range in apparent magnitude in the cloud is small another set of comparison stars that are brighter as well as one fainter will give larger relative parallaxes. If clouds would occur frequently in definite regions of the sky it would appear that the largest of the values for a given parallax is generally the most trustworthy. If two observatories use sets of comparison stars of the same mean magnitude the chances are that they will be both nearby, or both distant stars. The agreement between relative parallaxes for which $\bar{m}$, the mean magnitude of the comparison stars, is equal should, therefore, be expected to be better than for $\bar{m}$ not equal. For $\bar{m}$ not equal, however, one cannot predict which will go with the larger and which with the smaller parallax.

The only difficulty in this approach is that for a significant effect the parallaxes of the iIth magnitude stars would have to be larger than found from the statistical mean parallaxes. This is, however, very difficult to accept and the suggestion has not been taken seriously. The statistical method came to the fore when the distribution of densities and velocities was believed to be isotropic. A number of recent discoveries concerning the spiral structure have made serious inroads into the idea of isotropic distribution even within a few hundred parsecs from the sun. The analysis of proper motions and radial velocities by means of spherical harmonics and other methods whereby the proper motions in one part of the sky are assumed to correspond to the radial velocities in another are at present less convincing than before. An independent method to determine the mean parallax of faint stars may be the following. Assuming that the spectroscopic criteria are known, a sufficiently large number of stars within narrow limits of absolute magnitude may be picked out from the available trigonometric parallax material. By plotting the relative parallaxes as ordinates against the photometric ones as abscissae the $y$-intercept of a straight line, which may be determined by least squares, will represent the mean parallax of the comparison stars. The slope will at the same time give the correction to the scale of the photometric parallaxes. The reliability of this method depends above all on the constancy of the mean absolute magnitude with respect to apparent magnitude. If this mean constancy can be guaranteed the dispersion in absolute magnitude is 
not very important, certainly need not be less than a few tenths. The statistical regression, if noticeable, will tend to decrease the slope and the size of the intercept, making the mean parallax of the comparison stars too small.

A preliminary test was carried through for extensive material, and the result as shown below seems to be quite in accordance with the expectation. Faint stars of small proper motion offer little chance of discovery, and their existence can best be investigated by the observation of radial velocities for a large number of stars around magnitude I I.5. Such observations would at the same time serve for a better knowledge of the solar motion relative to faint stars (which is urgently needed).

The mean diameter of the comparison stars was taken, for the present test, from the following sources: Yale, Volumes 8 and I5; Greenwich, First and Second Parallax Volumes; Cape, Ist, 2nd, 6th, 7th and 8th Series; Allegheny, practically all up to the list in $A . J$. 49. Only parallaxes observed with photographic refractors for which the mean magnitudes of the comparison stars were available are included. The last three Cape series $(6,7$ and 8$)$ contain many parallaxes not in the 1952 Parallax Catalogue which have also been used.

The following numbers of parallaxes common to two stations, for which $\bar{m}$ was found for both in the above sources, are available: Y-C I69; A-Y III; A-G 48; A-C I4. The statistics in Table II are for $\Delta \bar{m}<0.3$ and $\Delta \bar{m} \geqslant 0.3$, and for a subdivision of the latter group.

The mean differences o".or 8 and o".02 I show a gain which corresponds to a mean error o".oro. The limit within which lie 50 per cent of the differences shows a gain of $\left(0.014^{2}-0.01 I^{2}\right)^{\frac{1}{2}}$ or almost o".oog, and the gain is, therefore, chiefly in the smaller differences, as is also indicated by the percentages smaller than o".oro.

The systematic differences for the two cases that have a sufficient number of stars are compared with the Precepts of the 1935 and $195^{2}$ catalogues below:

$$
\begin{array}{llcc} 
& & \text { 1935 } & \text { 1952 } \\
\text { A-Y } & \text {-o".005 (I I I ) } & - \text { o".003 } & \text {-o".005 } \\
\text { C-Y } & \text {-o".00I (I69) } & - \text { o".002 } & \text { +o".00I }
\end{array}
$$

TABLE II. COMPARING PARALLAXES OF EQUAL AND UNEQUAL COMPARISON STARS

\begin{tabular}{|c|c|c|}
\hline & $\Delta \bar{m}<0.3$ & $\Delta \bar{m} \geqslant 0.3$ \\
\hline r.m.s. difference & o".oI 8 & $0 " .021$ \\
\hline $50 \%$ limit & o".oI I & o".oI 4 \\
\hline$\%<$ o".oro & $47 \%$ & $34 \%$ \\
\hline No. of pairs & IO2 & 240 \\
\hline \multirow{3}{*}{$\begin{array}{l}A-Y \\
C-Y\end{array}$} & $-0.0003(45)$ & -0 ".0082 (66) \\
\hline & -0".0022 (37) & -0".00II (I32) \\
\hline & $0.3 \leqslant \Delta \bar{m}<0.6$ & $\Delta \bar{m} \geqslant 0.6$ \\
\hline r.m.s. difference & o".020 & o".022 \\
\hline $50 \%$ limit & o".or4 & o".or4 \\
\hline$\%<$ o".oro & $34 \%$ & $34 \%$ \\
\hline No. of pairs & 92 & 148 \\
\hline$A-Y$ & -o".0092 (26) & -0 ".0075 (40) \\
\hline $\mathrm{C}-\mathrm{Y}$ & -o".ooI I (48) & -o".00I I (84) \\
\hline
\end{tabular}

The systematic differences for these same stations are also given for small and large $\Delta \bar{m}$ separately in Table II. That between Cape and Yale which has opposite signs in the two catalogues is not significant, and the value -0 ".0022 in Table II is due to one or two large differences. The systematic difference between Allegheny and Yale is perhaps the best known, and it has caused more concern than other cases because both series were started and for a long time operated along the same lines by Schlesinger. The present sample of I I I stars gives A-Y = -0 ".005 which is also the value derived by Miss Jenkins, but this is, as shown in Table II, entirely due to the stars for which $\Delta \bar{m} \geqslant 0.3$.

Whereas the present test does not yet prove the existence of nearby clouds of small proper motion stars, it seems to justify a renewed interest in the mean parallax of the comparison stars.

REFERENCES

I. General Catalogue of Trigonometric Parallaxes, $195^{2}$.

2. A. J. 53, 23I, I948.

3. Oliver J. Lee, Ann. Dearborn Obs. 4, I, 1930; W. J. Luyten, J. Schilt and P. van de Kamp, Pop. Astr. 56, 423,1948 .

Rutherfurd Observatory, Columbia University, New York, N.Y. 1953 September. 